motif, while the C-terminal domain shows a typical restriction endonuclease fold. By structural comparison and mutational analysis we showed that the active site of SdaI is located at the C-terminal domain and exhibits a new variation of the canonical PD...(D/E)XK active site motif. Mutational analysis of the residues from the predicted recognition helix of the wHTH motif suggests that SdaI determinants of sequence specificity are clustered at the N-terminal domain. The modular architecture of SdaI, wherein one domain mediates DNA binding while the other domain is predicted to catalyze hydrolysis, distinguishes SdaI from the previously characterized restriction enzymes interacting with symmetric recognition sequences.

[1] Roberts RJ, Belfort M, Bestor T, Bhagwat AS, Bickle TA, Bitinaite J, Blumenthal RM, et al. Nucleic Acids Res. 2003, 31, 1805.

[2] Grazulis, S., Manakova, E., Roessle, M., Bochtler, M., Tamulaitiene, G., Huber, R., Siksnys, V. Proc. Natl. Acad. Sci. USA, 2005, 102, 15797.

[3] Pingoud, A., Fuxreier, M., Pingoud, V., Wende, W., Cell Mol. Life Sci., 2005, 62, 685.

\section{MS04 P08}

C1-inhibitor structure reveals a novel mechanism of heparin potentiation Veronika Harmat ${ }^{\mathrm{a}, \mathrm{b}}$, László Beinrohr $^{\mathrm{c}}$, József Dobóc ${ }^{\mathrm{c}}$ Zsolt Lőrincz ${ }^{\mathrm{c}}$, Péter Gál ${ }^{\mathrm{c}}$, Gábor Náray-Szabóa, ${ }^{\mathrm{a}, \mathrm{b}}$, Péter Závodszky ${ }^{\mathrm{c}}{ }^{a}$ Structural Biology and Chemistry Laboratory, Institute of Chemistry, Eötvös Loránd University, Budapest, Hungary. ${ }^{b}$ Protein Modelling Group, Eötvös Loránd University, Hungarian Academy of Sciences, Budapest, Hungary. ${ }^{c}$ Institute of Enzymology, Biological Research Center, Hungarian Academy of Sciences, Budapest,Hungary. E-mail: harmatv@ludens.elte.hu

\section{Keywords: immune regulation, serine-protease inhibitors, electrostatic interactions}

C1-inhibitor (C1-inh), a member of the serpin family of serine-protease inhibitors, is the major downregulator of inflammatory processes in blood plasma. Genetic deficiency of C1-inh results in hereditary angioedema, a dominantly inheritable, potentially lethal disease. Antiinflammatory activity of heparin a naturally occurring glycosaminoglycan is realized through increasing inhibitory activity of C1-inh.

Activities of many serpins are modulated by ligand binding. Heparin enhances the inhibitory effect of antithrombin by "bridging" the two proteins. Allostery also plays an important role in the heparin activation of antithrombin. Polyanion potentiation of protein $\mathrm{C}$ inhibitor against protein $\mathrm{C}$ by the "co-occupation" mechanism is also based on migration of proteins along the heparin chain. These mechanisms fail to explain the effect of heparin on C1-inh activity.

We crystallized the serpin domain of C1-inh using the hanging drop method. Collection of diffraction data was carried out at $100 \mathrm{~K}$ on beamline X11 of EMBL-outstation at DESY, Hamburg, Germany. The structure was determined using molecular replacement and refined to $2.35 \AA$ resolution.

The structure represents a novel latent serpin form with 7stranded sheet $\mathrm{A}$ and a truncated disordered $\mathrm{N}$-terminal domain, which explains functional consequences of the numerous naturally occurring mutations. Unique conformation of the C-terminal tail indicates its unexpected role as a barrier in the active-latent transition. On the basis of surface charge pattern, heparin affinity measurements and docking of a heparin disaccharide, a heparin binding site is proposed in the contact area of the serpin-proteinase encounter complex. We show how polyanions change selectivity of $\mathrm{C} 1$-inh by a novel "sandwich" mechanism, explaining earlier reaction kinetic and mutagenesis data. These results provide the explanation of heparin's antiinflammatory activity and may help improving therapeutic $\mathrm{C} 1$-inh preparations in treatment of common inflammatory diseases, such as organ transplant rejection and heart attack.

\section{MS04 P09 \\ Crystal structures of flax rust avirulence proteins AvrL567-A and AvrL567-D. Gregor Guncar ${ }^{\mathrm{a}, \mathrm{b}, 1,2}$, Ching-I A. Wang, ${ }^{\mathrm{a}, 1}$, Jade K. Forwood ${ }^{\mathrm{a}, \mathrm{b}}$, Trazel Teh ${ }^{\mathrm{a}}$, Ann-Maree Catanzariti ${ }^{\mathrm{d}}$, Gregory J. Lawrence ${ }^{\mathrm{c}}$, Horst Joachim Schirra $^{\mathrm{b}}$, Peter A. Anderson ${ }^{\mathrm{e}}$, Jeffrey G. Ellis ${ }^{\mathrm{c}}$, Peter N. Dodds ${ }^{c}$ and Bostjan Kobe ${ }^{\mathrm{a}, \mathrm{b}, \mathrm{f}}$. ${ }^{a}$ School of Molecular and Microbial Sciences, University of Queensland(UQ), Brisbane, Australia ${ }^{b} I M B, \quad U Q$, Brisbane, Australia ${ }^{c}$ Division of Plant Industry, CSIRO, Canberra, Australia ${ }^{d}$ Department of Plant and Microbial Biology, University of California, Berkeley, USA ${ }^{e} S B S$, Flinders University of South Australia, Adelaide, Australia ${ }^{f} S R C$ for Functional and Applied Genomics, UQ, Brisbane, Australia ${ }^{I}$ These authors contributed equally to this work. ${ }^{2}$ On leave from Josef Stefan Institute, Ljubljana, Slovenia.. E-mail: g.guncar@uq.edu.au}

\section{Keywords: avirulence, plant resistance, SAD}

Plants have evolved a versatile multi-layered defence system to fight pathogens. The gene-for-gene model of plant disease resistance involves direct or indirect recognition of pathogen avirulence (Avr) proteins by plant resistance (R) proteins. Flax rust (Melampsora lini) AvrL567 avirulence proteins and the corresponding flax (Linum usitatissimum) L5, L6 and L7 resistance proteins interact directly. We determined the three-dimensional structures of two members of the AvrL567 family, AvrL567-A and AvrL567-D. The structure of AvrL567-A was solved by single-wavelength anomalous dispersion (SAD) technique, using the dataset collected on the conventional in-house $\mathrm{Cu} \mathrm{X}$-ray generator, taking advantage of a bound $\mathrm{Co}^{2+}$ ion. The structure of AvrL567$\mathrm{D}$ was solved by molecular replacement, using the structure of AvrL567-A as a search model. The structures of both proteins are very similar and reveal a $\beta$-sandwich fold with no close known structural homologues. The polymorphic residues in the AvrL567 family map to the surface of the protein and polymorphisms in residues associated with recognition differences lead to significant changes in surface chemical properties. Analysis of single amino acid substitutions in AvrL567 proteins confirm the role of individual residues in conferring differences in recognition and suggest that recognition specificity of the L proteins for the AvrL567 family members results from the cumulative effects of multiple amino acid contacts. The structure also provides insights into possible pathogen-associated functions of AvrL567 proteins, with nucleic acid binding activity demonstrated in vitro, suggesting a possible role in influencing host gene expression during infection. Our studies provide some of the first structural information on avirulence proteins that 